\title{
FREE IDEALS IN RINGS OF FUNCTIONS
}

E. S. WOLK

1. Introduction. Let $X$ be any infinite set, and $R$ a ring with unit element $e$. Let $A(X, R)$ be the ring of all functions from $X$ to $R$, with the usual definitions of + and $\cdot$. No topological considerations are introduced; i.e., all the sets involved are taken as discrete. Let $I$ be an ideal in $A$. Following Hewitt [2] and Kaplansky [3], we say that $I$ is free if and only if for each $x \in X$ there exists $f \in I$ such that $f(x)=e$. The purpose of this paper is to give an exact characterization of all the free left ideals of $A$. The results take a particularly simple form if we make the additional assumption that every left ideal in $R$ is principal.

2. Preliminary definitions and lemmas. Let us denote by $L$ the set of all left ideals of $R$. We shall consider $L$ as a lattice under the usual operations of + and $\cap$. We admit $\{0\}$ and $R$ as elements of $L$.

We denote by $L^{\boldsymbol{x}}$ the set of all functions from $X$ to $L$. If $p \in L^{X}$, $q \in L^{X}$, we define $p=q$ to mean that $p(x)=q(x)$ for all but a finite number of $x \in X$. We define $p+q$ by $(p+q)(x)=p(x)+q(x)$, and $p \cap q$ by $(p \cap q)(x)=p(x) \cap q(x)$. Under these operations $L^{x}$ becomes a lattice, in which $p<q$ means that $p(x) \subset q(x)$ for all but a finite number of $x \in X$. The function in $L^{X}$ which is identically 0 will be denoted by $\theta$. For each $p \in L^{\boldsymbol{X}}$ we define $\mu(p)=\{x \in X \mid p(x) \neq R\}$.

The set of all subsets of $X$ will be denoted by $2^{x}$. If $\alpha \in 2^{x}, \beta \in 2^{x}$, we define $\alpha=\beta$ to mean that $\alpha$ and $\beta$ are identical save for a finite set of points. We denote the empty set by $\varnothing$. Thus $\alpha=\varnothing$ means that $\alpha$ is a finite subset of $X$. We consider $2^{X}$ as a lattice under the usual operations of $U$ and $\cap$. In this lattice $\alpha \subset \beta$ means that all but a finite number of points of $\alpha$ lie in $\beta$.

The set of all free left ideals of $A(X, R)$ will be denoted by $F(A)$.

The proof of the following lemma may be left to the reader.

Lemma 1. Let $x_{1}, x_{2}, \cdots, x_{n}$ be a finite number of points of $X$, and let $a_{1}, a_{2}, \cdots, a_{n}$ be arbitrary elements of $R$. Then for any $I \in F(A)$ there exists $f \in I$ such that $f\left(x_{i}\right)=a_{i}$ for $i=1,2, \cdots, n$, and $f(x)=0$ for all other $x \in X$.

Let us write

$$
J_{0}=\{f \in A \mid f(x)=0 \text { for all but a finite number of } x \in X\} .
$$

Received by the editors May 12, 1954 and, in revised form, December 20, 1954. 
Clearly $J_{0} \in F(A)$. If $I$ is any left ideal in $A$, Lemma 1 implies that $I \in F(A)$ if and only if $I \supset J_{0}$. Thus the intersection of any number of ideals in $F(A)$ is again an ideal in $F(A)$. Also it is obvious that $I_{1} \in F(A)$ and $I_{2} \in F(A)$ imply $I_{1}+I_{2} \in F(A)$. Hence $F(A)$ is a lattice with respect to + and $\cap$, and the ideal $J_{0}$ is its 0 element.

For $\alpha \in 2^{x}, p \in L^{x}$, we now define

$$
J(\alpha, p)=\{f \in A \mid f(x) \in p(x) \text { for all but a finite number of } x \in \alpha\},
$$

with the agreement that $J(\varnothing, p)=A$ for all $p$. It is clear that $J(\alpha, p)$ $\in F(A)$, and that $J(X, \theta)=J_{0}$. The following facts are also obvious.

LEMMA 2.

(1) $J(\alpha, p)=A$ if and only if $\mu(p) \cap \alpha=\varnothing$.

(2) $J(\alpha, p) \subset J(\beta, p)$ if and only if $\beta \subset \alpha$.

(3) $J(\alpha, p) \subset J(\alpha, q)$ if and only if $p(x) \subset q(x)$ for all but a finite number of $x \in \alpha$.

Lemma 3. $J(\alpha, p)+J(\beta, q)=J(\alpha \cap \beta, p+q)$.

Proof. Suppose $f \in J(\alpha, p), g \in J(\beta, q)$. Then for all but a finite number of $x \in \alpha \cap \beta$, we have $f(x)+g(x) \in p(x)+q(x)$. Hence $J(\alpha, p)$ $+J(\beta, q) \subset J(\alpha \cap \beta, p+q)$. Conversely, suppose that $f \in J(\alpha \cap \beta, p+q)$. We define functions $f_{1}$ and $f_{2}$ in $A$ as follows:

$$
\begin{aligned}
& f_{1}=0 \text { on the complement of } \alpha \cup \beta, \\
& f_{1}=f \text { on } \beta \cap \alpha^{\prime}, \\
& f_{1}=0 \text { on } \alpha \cap \beta^{\prime}, \\
& f_{2}=f \text { on the complement of } \alpha \cup \beta, \\
& f_{2}=0 \text { on } \beta \cap \alpha^{\prime}, \\
& f_{2}=f \text { on } \alpha \cap \beta^{\prime} .
\end{aligned}
$$

For $x \in \alpha \cap \beta$ we have $f(x)=a_{x}+b_{x}$, where $a_{x} \in p(x)$ and $b_{x} \in q(x)$ for all but a finite number of $x \in \alpha \cap \beta$. Thus for $x \in \alpha \cap \beta$, we define $f_{1}(x)=a_{x}, f_{2}(x)=b_{x}$. Then $f_{1} \in J(\alpha, p), f_{2} \in J(\beta, q)$, and $f=f_{1}+f_{2}$. Hence $J(\alpha \cap \beta, p+q) \subset J(\alpha, p)+J(\beta, q)$.

Now for $f \in A$, let us write

$$
\begin{aligned}
& \sigma(f)=\{x \in X \mid f(x) \text { has no left inverse }\}, \\
& \lambda(f)=\text { complement of } \sigma(f) .
\end{aligned}
$$

Also, for $f \in A$, we define $p_{f} \in L^{x}$ by

$$
p_{f}(x)=[f(x)]=\text { left ideal generated by } f(x) .
$$

Lemma 4. If $I \in F(A)$ and $f \in I$, then $J\left(\sigma(f), p_{f}\right) \subset I$.

Proof. Suppose $g \in J\left(\sigma(f), p_{f}\right)$. Then $g(x) \in p_{f}(x)$ for all $x \in \sigma(f)$ 
except for a finite set of points $x_{1}, x_{2}, \cdots, x_{n}$. I.e., for each $x \in \sigma(f)$, $x \neq x_{i}$, there exists $a_{x} \in R$ such that $g(x)=a_{x} f(x)$. For $x \in \lambda(f)$, let $f^{-1}(x)$ denote any left inverse of $f(x)$. Let us define a function $g_{1}$ in $A$ as follows:

$$
\begin{aligned}
& g_{1}(x)=a_{x} \text { for } x \in \sigma(f)-\left\{x_{1}, x_{1}, \cdots, x_{n}\right\}, \\
& g_{1}(x)=0 \text { for } x=x_{i}, i=1,2, \cdots, n, \\
& g_{1}(x)=g(x) \cdot f^{-1}(x), \text { for } x \in \lambda(f) .
\end{aligned}
$$

Then for $i=1,2, \cdots, n$ we have $\left(g_{1} f\right)\left(x_{i}\right)=0$, and $\left(g_{1} f\right)(x)=g(x)$ for all other $x \in X$. But by Lemma 1 , there exists $h \in I$ such that $h\left(x_{i}\right)$ $=g\left(x_{i}\right)$ for $i=1,2, \cdots, n$, and $h(x)=0$ for all other $x$. Then $g=g_{1} f$ $+h \in I$.

3. Structure of the free left ideals of $A(X, R)$. Following Birkhoff $[1$, p. 21], we introduce the following definitions.

Definition. A subset $K$ of $L^{x}$ is an ideal in $L^{x}$ if and only if

(1) $p \in K$ and $q \in K$ imply $p+q \in K$,

(2) $p \in K$ and $q<p$ imply $q \in K$.

The set of all ideals of $L^{X}$ will be denoted by $K(X, R)$. We admit $\{\theta\}$ and $L^{X}$ as elements of $K(X, R)$.

Definition. A subset $D$ of $2^{x}$ is a dual ideal in $2^{x}$ if and only if

(1) $\alpha \in D$ and $\beta \in D$ imply $\alpha \cap \beta \in D$,

(2) $\alpha \in D$ and $\beta \supset \alpha$ imply $\beta \in D$.

The set of all dual ideals of $2^{x}$ will be denoted by $\mathcal{D}(X)$. We admit $\{X\}$ and $2^{X}$ as elements of $\mathcal{D}(X)$.

Now for $D \in \mathcal{D}(X)$ and $K \in K(X, R)$ we define

$$
J(D, K)=\underset{\alpha \in D, p \in K}{\bigcup} J(\alpha, p),
$$

where the "U" denotes the set-theoretic union of the $J(\alpha, p)$. We verify that $J(D, K) \in F(A)$. Suppose that $f$ and $g$ are functions in $J(D, K)$. Then there exist $\alpha$ and $\beta$ in $D$, and $p$ and $q$ in $K$, such that $f \in J(\alpha, p)$ and $g \in J(\beta, q)$. By Lemma $3, f+g \in J(\alpha \cap \beta, p+q)$ $\subset J(D, K)$, from which it follows that $J(D, K)$ is a left ideal.

Also note that $J(D, K)=A$ implies that the function which is identically equal to $e$ is in $J(\alpha, p)$ for some $\alpha \in D$ and $p \in K$; from this it follows that $J(D, K)=A$ if and only if $J(\alpha, p)=A$ for some $\alpha \in D$, $p \in K$.

Lemma 5. $I \in F(A)$ implies $I=\bigcup_{\alpha \in D, \vartheta \in I} J\left(\alpha, p_{\vartheta}\right)$ for some $D \in \mathcal{D}(X)$.

Proof. Define $D=\{\sigma(f) \mid f \in I\}$. (It is obvious that $D=2^{x}$ if and only if $I=A$.) We show that $D \in \mathcal{D}(X)$. Clearly, $\alpha \in D$ and $\gamma \supset \alpha$ imply 
$\gamma \in D$. (Use the function which is 0 on $\gamma$ and $e$ on $X \cap \gamma^{\prime}$.) Now suppose $\alpha \in D, \beta \in D, \alpha=\sigma(f), \beta=\sigma(g)$, where $f, g \in I$. Let us define $f^{*} \in A$ and $g^{*} \in A$ as follows:

$$
\begin{aligned}
& f^{*}(x)=\text { any left inverse of } f(x), \text { for } x \in \lambda(f), \\
& f^{*}(x)=0 \text { for } x \in \sigma(f), \\
& g^{*}(x)=0 \text { for } x \in \sigma(g), \\
& g^{*}(x)=\text { any left inverse of } g(x), \text { for } x \in \lambda(g) \cap \sigma(f), \\
& g^{*}(x)=0 \text { for } x \in \lambda(g) \cap \lambda(f) .
\end{aligned}
$$

Then $\left(f^{*} f+g^{*} g\right)(x)=0$ for $x \in \sigma(f) \cap \sigma(g)$, and $\left(f^{*} f+g^{*} g\right)(x)=e$ for all other $x \in X$. Hence $\sigma\left(f^{*} f+g^{*} g\right)=\sigma(f) \cap \sigma(g)=\alpha \cap \beta$. But $f^{*} f+g^{*} g \in I$. Hence $\alpha \cap \beta \in D$, and it follows that $D \in \mathcal{D}(X)$.

Now let $f$ and $g$ be arbitrary functions in $I$. By Lemma $4, J\left(\sigma(f), p_{f}\right)$ $\subset I, J\left(\sigma(g), p_{g}\right) \subset I$; and hence by Lemma 3, $J\left(\sigma(f) \cap \sigma(g), p_{f}+p_{g}\right) \subset I$. Using (2) and (3) of Lemma 2, we then have

$$
J\left(\sigma(f), p_{g}\right) \subset J\left(\sigma(f) \cap \sigma(g), p_{g}\right) \subset J\left(\sigma(f) \cap \sigma(g), p_{f}+p_{g}\right) \subset I,
$$

and hence $\bigcup_{\alpha \in D, g \in I} J\left(\alpha, p_{g}\right) \subset I$. Since it is obvious that we also have $I \subset \bigcup_{\alpha \in D, g \in I} J\left(\alpha, p_{g}\right)$, the lemma is proved.

We are now ready for our main result.

THEOREM 1. Let $R$ be a ring with unit in which each left ideal is principal, and let $A$ be the ring of all functions from $X$ to $R$. Then $I \in F(A)$ if and only if $I=J(D, K)$ for some $D \in \mathcal{D}(X)$ and $K \in K(X, R)$.

Proof. Define $D$ as in Lemma 5. Let $K=\left\{p_{\theta} \mid g \in I\right\}$. We show that $K \in K(X, R)$. First suppose that $p_{\theta} \in K$ and $q<p_{g}$. Then $q(x) \subset p_{g}(x)$ for all $x \in X$ save for a finite set of points $x_{1}, x_{2}, \cdots, x_{n}$. Since $g(x)$ generates the ideal $p_{g}(x)$, then for $x \neq x_{i}, i=1,2, \cdots, n$, there exists $m_{x} \in R$ such that $m_{x} g(x)$ generates the ideal $q(x)$. Define a function $f_{1} \in A$ by $f_{1}(x)=m_{x}$ for $x \neq x_{i}$, and $f_{1}\left(x_{i}\right)=0$ for $i=1,2, \cdots, n$. Let $a_{i}$ be an element of $R$ which generates the ideal $q\left(x_{i}\right)$. By Lemma 1 , there exists $f_{2} \in I$ such that $f_{2}\left(x_{i}\right)=a_{i}$ for $i=1,2, \cdots, n$, and $f_{2}(x)=0$ for all other $x \in X$. Then $f=f_{1} g+f_{2} \in I$, and $p_{f}=q$. Hence $q \in K$.

Now suppose that $g \in I$ and $h \in I$. For each $x \in X$, let $c_{x}$ be an element of $R$ which generates the ideal $p_{\theta}(x)+p_{h}(x)$. Let $f$ be the function in $A$ such that $f(x)=c_{x}$ for all $x$. Then $p_{f}=p_{g}+p_{h}$. But $f \in J(\sigma(g)$ $\left.\cap \sigma(h), p_{g}+p_{h}\right) \subset I$. Hence $p_{g}+p_{h} \in K$, and $K \in K(X, R)$. The theorem now follows from Lemma 5.

4. A special case. In the special case when $R$ is a division ring, the above discussion is of course greatly simplified; and we can also easily obtain an abstract characterization of the lattice $F(A)$. Assuming now that $R$ is a division ring, we define, for $\alpha \in 2^{x}$, 


$$
J(\alpha)=\{f \in A \mid f(x)=0 \text { for all but a finite number of } x \in \alpha\},
$$

with the agreement that $J(\varnothing)=A$. The following relations are easily verified.

Lemma 6. For $\alpha \in 2^{x}, \beta \in 2^{x}$, we have

$$
\begin{aligned}
& J(\alpha)+J(\beta)=J(\alpha \cap \beta), \\
& J(\alpha) \cap J(\beta)=J(\alpha \cup \beta) .
\end{aligned}
$$

For $D \in \mathcal{D}(X)$, we now define $J(D)=\bigcup_{\alpha \in D} J(\alpha)$. We then obtain the following form of Theorem 1, making the appropriate simplifications in the proof. This result has, in essence, already been obtained by Hewitt [2, Theorem 36]. We omit the details.

TheOREм $1^{\prime}$. Let $R$ be a division ring, $A$ the ring of all functions from $X$ to $R$. Then $I \in F(A)$ if and only if $I=J(D)$ for some $D \in \mathcal{D}(X)$.

In this special case we now show how to construct from the set $X$ a lattice-isomorphic image of the lattice $F(A)$. First we prove

LEMMA 7. $J\left(D_{1}\right)=J\left(D_{2}\right)$ if and only if $D_{1}=D_{2}$.

Proof. Suppose $J\left(D_{1}\right)=J\left(D_{2}\right)$, and $\alpha \in D_{1}$. The function $f$ which is 0 on $\alpha$ and $e$ on the complement of $\alpha$ is in $J(\alpha) \subset J\left(D_{1}\right)$. Then $f \in J(\beta)$ for some $\beta \in D_{2}$. This means $\alpha \supset \beta$, whence $\alpha \in D_{2}$. Hence $D_{1} \subset D_{2}$, and likewise $D_{2} \subset D_{1}$.

Now for $D_{1} \in \mathcal{D}(X), D_{2} \in \mathcal{D}(X)$, we define

$$
\begin{aligned}
& D_{1} \cap D_{2}=\text { set-theoretic intersection of } D_{1} \text { and } D_{2}, \\
& D_{1}+D_{2}=\left\{\gamma \in 2^{x} \mid \gamma \supset \alpha \cap \beta \text { for some } \alpha \in D_{1} \text { and } \beta \in D_{2}\right\} .
\end{aligned}
$$

It is easily verified that $\mathcal{D}(X)$ forms a lattice under these operations. We then obtain the following theorem, the proof of which will be left to the reader.

Theorem 2. Let $R$ be a division ring, and $A$ the ring of all functions from $X$ to $R$. Then the correspondence $J(D) \leftrightarrow D$ is a lattice-isomorphism of $F(A)$ with $\mathcal{D}(X)$.

\section{REFERENCES} 1948.

1. G. Birkhoff, Lattice theory, Amer. Math. Soc. Colloquium Publications, vol. 25,

2. E. Hewitt, Rings of real-valued continuous functions, Trans. Amer. Math. Soc. vol. 64 (1948) pp. 45-99.

3. I. Kaplansky, Topological rings, Amer. J. Math. vol. 69 (1947) pp. 153-183.

UNIVERSITY OF CONNECTICUT 